\title{
DAMPING RATIO ESTIMATION USING HYBRID METHODS
}

\author{
Cheng-Yuan Liou and Chien-Chin Shen \\ Department of Computer Science and Information Engineering, National Taiwan University, \\ Taipei, Taiwan 10764, Republic of China
}

(Received 3 January 1988, accepted 28 April 1989)

\begin{abstract}
Much effort has been expended in attempts to estimate dynamic response parameters of structures. Damping ratios have been estimated with varying degrees of success. Some of this effort has been motivated by active research and development of structural integrity monitoring systems, for design purposes, accurate estimates of damping ratios are important.

In this work we apply several modern spectral analysis methods (maximum likelihood method, maximum entropy method and the Lagunas-Hernandez method) plus the spectral moment relations near natural frequency to the estimation of damping ratios. We use simulated vibration data and test the different choices of the spectral methods in order to obtain the best estimation procedure.

The spectral shape of the response near the natural frequency is estimated first as the basis for further analysis. Half-power bandwidth and spectral moments are then derived from the spectral shape. The bandwidth and moments are subsequently related to the damping ratio. An iterated method for obtaining the damping ratio from the spectral moments has been proposed by Shyam Sunder. The half-power bandwidth and moments are crucial to this method as is the estimation of the spectral shape, there are many methods available to estimate spectral shape. Different combinations of the two methods gives varied damping ratios, in this work we try to identify the best combinations. We have used many sets of exact and simulated data and applies many different combinations. Results show that the maximum entropy spectral method and the method of Shyam Sunder is the best combination from our tests.
\end{abstract}

\section{INTRODUCTION}

There are many analytical [1] and experimental methods [2] for modal damping ratio identification which can be applied to diverse subjects [3]. In this work we focus on methods which identify the damping ratio by using the estimated spectrum and the estimated spectral moments.

There are many different computational methods for estimating the power spectral density (PSD) function using finite length data. We briefly review some important PSD function estimates. There are two major conventional methods those of Blackman and Tukey and Cooley and Tukey. The Blackman-Tukey method estimates the PSD function indirectly. In their indirect method the autocorrelation functions are estimated from the finite length data and then they are transformed by applying the Fourier transformation to obtain the PSD function. The Cooley-Tukey method is based on computing the PSD function via a finite-length fast Fourier transform (FFT) of the original data. This method can take full advantage of the FFT $[4,5]$. Both methods involve using finite length window functions. The performances of many variant methods depends critically on the choice of the length and shape of the windows.

Burg has devised the maximum entropy method (MEM) for the PSD estimation [6]. The data are modelled as an auto-regression (AR) model in the MEM. The MEM has high resolution ability in determining the PSD shape in Fougere [7] and it also gives a 
mathematically tractable model for random vibrations. Vandiver and Campbell used both simulated and measured data to study the MEM's performance on dynamic random vibrations. The results in refs $[7,8]$ reveal that the bias and the variance of the conventional damping estimator are strongly related to the properties of the spectral estimators. They also show that conventional damping estimation is not efficient for short length and noisy measurements. The use of such an inefficient damping estimator limits the utility of the damping ratio estimator. Comparisons of the MEM and conventional methods are available in their work, which proves that the MEM performs better in damping ratio estimation than the conventional methods.

We still lack knowledge about the relative performances of several other contemporary comparable modern PSD estimators, such as the maximum likelihood method (MLM) [6] and the Lagunas-Hernandez method [9]. They all use the autocorrelation functions to estimate the PSD functions, this motivated the present work. The MLM is derived from the minimum variance unbiased spectral estimator. The Lagunas-Hernande $\angle$ method is an improved version of the MLM. The performances of the MLM and the LagunasHernandez method in the spectral estimation shows that they hold promise for damping ratio estimation, in this work we apply MEM, MLM and Lagunas-Hernandez methods in estimating damping ratios and discuss their performance.

The data we use for comparing the performances of the three methods are created by both theoretical and estimated autocorrelation functions. This is because all three methods use the estimated autocorrelation functions.

The theoretical autocorrelation functions $[10,11]$ for a single-degree-of-freedom (sdof) dynamic system with a white random input is given as follows

$$
R_{x}(\tau)=\frac{\pi S_{0}}{2 \omega_{n}^{3} \beta} \exp \left(-\beta \omega_{n}|\tau|\right) \cdot\left\{\cos \left(\omega_{d}|\tau|\right)+\frac{\omega_{n} \beta}{\omega_{d}} \sin \left(\omega_{d}|\tau|\right)\right\}, \quad \omega_{d}=\omega_{n}\left(1-\beta^{2}\right)^{1 / 2}
$$

where $R_{x}(\tau)=E[x(t) x(t+\tau)]$ is the autocorrelation function at lag $\tau, \omega_{n}$ the undamped natural frequency, $\omega_{d}$ the damped natural frequency, $\beta$ the damping ratio, and $S_{0}$ the constant power over all frequencies of the white random input.

The estimated autocorrelation functions can be obtained from the simulated displacement data $\{x(t), t=0$ to $T(=N \Delta)\}$, where $\Delta$ is the sampling interval. The effects of the finite data length is important for damping ratio estimation. In order to generate a sequence of responses in the simulation, we use a "discrete analog" [12] technique to simulate the linear stationary dynamic system and use discrete Gaussian white noise as random input $\{u(n), n=0,1,2, \ldots$, where $t=n \cdot \Delta\}$. The "discrete analog" technique synthesises a linear discrete system that is analogous with a linear continuous system. The difference equation of the discrete system is derived and programmed on a digital computer.

\section{SPECTRAL SHAPE ESTIMATION}

In this section we briefly review the three power spectral estimators.

The MEM can be viewed as fitting a special AR model with order $M$ to the finite discrete autocorrelation functions $\left\{R_{x}(m), m=0,1,2, \ldots, M\right\}$ where $m$ is the discrete lag. Instead of the theoretical autocorrelation functions (1), the estimated autocorrelation functions

$$
\left\{R_{x}(m)=R_{x}(-m)=\frac{1}{N} \sum_{n=0}^{N-|m|-1} x(n) x(n+m), \quad 0 \leqslant m \leqslant M\right\}
$$


will be used for further spectral estimation. The autocorrelation function can be also obtained by overlap-add sectioning and FFT techniques [5] operating on the simulated finite length response data $\{x(n), n=0,1,2, \ldots, N-1\}$. Its estimated spectrum is

$$
\begin{gathered}
S_{M E M}\left(f=\frac{\omega}{2 \pi}\right)=\frac{P_{M} \Delta}{\left|1+\sum_{m=1}^{M} a_{m} \exp (-j 2 \pi m f \Delta)\right|^{2}} \\
P_{M}=R_{x}(0)-\sum_{m=1}^{M} a_{m} R_{x}(m) ; \quad j=\sqrt{ }(-1)
\end{gathered}
$$

where $\Delta$ is the sampling interval and $a_{m}$ 's, $m=1,2, \ldots, M$, are obtained by solving the Yule-Walker equations in [4].

$$
\sum_{k=0}^{M} a_{k} R_{x}(m-k)= \begin{cases}P_{M}, & m=0 \\ 0, & m=1,2, \ldots, M\end{cases}
$$

or in the matrix form

$$
\left(\begin{array}{cccc}
R_{x}(0) & R_{x}(-1) & \cdots & R_{x}(-M) \\
R_{x}(1) & R_{x}(0) & \cdots & R_{x}(1-M) \\
\vdots & \vdots & & \vdots \\
R_{x}(M-1) & R_{x}(M-2) & \cdots & R_{x}(-1) \\
R_{x}(M) & R_{x}(M-1) & \cdots & R_{x}(0)
\end{array}\right)\left(\begin{array}{c}
1 \\
a_{1} \\
\vdots \\
a_{M-1} \\
a_{M}
\end{array}\right)=\left(\begin{array}{c}
P_{M} \\
0 \\
\vdots \\
0 \\
0
\end{array}\right) .
$$

Applying Levinson's iterative algorithm, we can solve the above Yule-Walker equation efficiently, see [4] for details. Now let

$$
R_{x}=\left(\begin{array}{cccc}
R_{x}(0) & R_{x}(-1) & \cdots & R_{x}(-M) \\
R_{x}(1) & R_{x}(0) & \cdots & R_{x}(1-M) \\
\vdots & \vdots & & \vdots \\
R_{x}(M-1) & R_{x}(M-2) & \cdots & R_{x}(-1) \\
R_{x}(M) & R_{x}(M-1) & \cdots & R_{x}(0)
\end{array}\right)
$$

The MLM is then given by the following formula [6]

$$
S_{M L M}(f)=\frac{1}{E^{T} R_{x}^{-1} E^{*}} \quad \text { where } E=\left[1, \mathrm{e}^{j 2 \pi f \Delta}, \mathrm{e}^{j 2 \pi f \Delta \cdot 2}, \ldots, \mathrm{e}^{j 2 \pi f \Delta \cdot(M)}\right]^{T}
$$

where ${ }^{*}$ denotes the complex conjugate operation and ${ }^{T}$ denotes the operation of transpose. $S_{M L M}(f)$ is the power which would come through a filter designed to pass frequency $f$ undistorted and to reject all other frequency components in an optimum manner. The computation of the above formula can be much reduced when the symmetric Toeplitz form of the matrix $R_{x}$ is considered.

The Lagunas-Hernandez spectral function [9] is given as in the following formula

$$
S_{L A G}(f)=\frac{E^{T} R_{x}^{-1} E^{*}}{E^{T} R_{x}^{-2} E^{*}} .
$$

The above formula is an improved MLM and is a true PSD function.

A major impediment to the wide-spread usage of these three spectral estimators has been the difficulty in selecting the order $M$ which produces the optimum estimate. Several criteria have been suggested as an aid in the selection process, such as final prediction-error criterion and information theoretical criterion [4], but as yet a definite general technique 
has not emerged. In the following discussion we detail a procedure which has a sufficiently small effect on the order selection for obtaining optimum natural frequency and damping ratio estimates.

\section{ALGORITHM FOR SPECTRAL MOMENTS}

In this section we briefly review two methods for the damping ratio estimation given the estimated PSD function, one is the iterative method of Sunder and the other is the traditional half-power method. The small damping ratio assumption is valid in all our work and we assume each mode of interest can be well resolved by the estimated PSD shape, we can then apply the dynamics of a sdof system near the maximum response frequency.

For a sdof dynamic system, whose FRF at frequency $\omega$ is $H(\omega)$, under white noise excitation and whose power spectral density is a constant $S_{0}$ at all frequencies, the theoretical displacement response spectral density $\left(S_{x}(\omega)=|H(\omega)|^{2} S_{0}\right)$ can be expressed as [11]

$$
S_{x}(\omega)=\frac{S_{0}}{\left(\omega_{n}^{2}-\omega^{2}\right)^{2}+4 \beta \omega_{n}^{2} \omega^{2}} \quad 0<\omega<\infty
$$

where $\omega_{n}$ and $\beta$ are time-invariant parameters.

We now review the iterative method, the first three spectral moments $\lambda_{i}, i=0,1,2$, are defined [13] as

$$
\lambda_{i}=\int_{\omega_{s}}^{\omega_{b}} \omega^{i} S_{x}(\omega) d \omega \quad i=0,1,2
$$

where $\omega_{a}$ and $\omega_{b}$ are lower and upper limits of the integrand respectively. The analytic expressions for these three moments are

$$
\lambda_{i}=\frac{S_{0} I_{i}}{4 \beta \omega_{n}^{3-i}} \quad i=0,1,2
$$

where

$$
I_{o}=\left.\left(D_{1}+D_{2}\right)\right|_{r_{1}^{2}} ^{r_{2}}, \quad I_{1}=\left.\frac{2}{d}\left(D_{3}\right)\right|_{r_{1}} ^{r_{2}}, \quad I_{2}=\left.\left(D_{1}-D_{2}\right)\right|_{r_{1}^{2}} ^{r_{2}} \quad 0 \leqslant I_{i}<\pi
$$

with

$$
\begin{aligned}
& D_{1}=\left[\tan ^{-1}\left(\frac{r-\sqrt{ }\left(1-\beta^{2}\right)}{\beta}\right)+\tan ^{-1}\left(\frac{r+\sqrt{ }\left(1-\beta^{2}\right)}{\beta}\right)\right], \\
& D_{2}=\frac{\beta}{d} \ln \left(\frac{1+r d+r^{2}}{1-r d+r^{2}}\right), \quad D_{3}=\tan ^{-1}\left(\frac{1-2 \beta^{2}-r^{2}}{-\beta d}\right)
\end{aligned}
$$

$d=2 \sqrt{ }\left(1-\beta^{2}\right)$, and $r_{1}, r_{2}$ are normalised integration limits which are defined as

$$
r_{1}=\frac{\omega_{a}}{\omega_{n}}, \quad r_{2}=\frac{\omega_{b}}{\omega_{n}} .
$$

Note that the quantities $\left\{D_{i}, i=1,2,3\right\}$ are functions of the damping ration $(\beta)$ and normalised frequencies $\left(r_{1}\right.$ and $\left.r_{2}\right)$, the quantities $\left\{I_{i}, i=0,1,2\right\}$ are also functions of the damping ratio $(\beta)$ and normalised frequencies $\left(r_{1}\right.$ and $\left.r_{2}\right)$. 
The average zero up-crossing frequency $\omega_{\text {up }}$ and Vanmarck's spectral bandwidth $\delta$ can be expressed as two functions of the three spectral moments [13] with the following identities

$$
\omega_{u p}=\sqrt{ }\left(\frac{\lambda_{2}}{\lambda_{0}}\right), \quad \delta^{2}=1-\frac{\lambda_{1}^{2}}{\lambda_{0} \lambda_{2}}, \quad \delta^{2}=1-\frac{I_{1}^{2}}{I_{0} I_{2}} \quad 0 \leqslant \delta \leqslant 1 .
$$

The theoretical relation between the natural frequency and the average zero up-crossing frequency is $\omega_{n}=\omega_{u p} \sqrt{ }\left(I_{0} / I_{2}\right)$. The concept of Vanmarck's spectral bandwidth can be seen from the approximated relation $\beta \approx(\pi / 4) \delta^{2}$.

Sunder devised an iterative algorithm $[14,15]$ to solve $\beta$ based on the above-formulae. Assume we know the maximum response frequency $\omega_{\max }$ of the mode under consideration from any of the three estimated PSD shapes using equations (2), (5). The algorithm is

Step 1: Guess an initial value for the damping ratio. This may be an arbitrarily small value, say $0 \cdot 001$.

Step 2: Compute the first three moments $\lambda_{0}, \lambda_{1}, \lambda_{2}$ of the calculated response PSD functions.

Step 3: Iterate to convergence on the function $F(\beta)$, it is given as

$$
F(\beta)=\frac{I_{1}^{2}}{I_{0} I_{2}}-\frac{\lambda_{1}^{2}}{\lambda_{0} \lambda_{2}}
$$

$F(\beta)$ depends on the variable $\beta$ only, and will be zero for the true value $\beta$. When we get the estimated $\beta$, the natural frequency can be estimated with the formula

$$
\omega_{n}=\omega_{u p} \sqrt{ }\left(\frac{I_{0}}{I_{2}}\right)
$$

Three suggested options of the integration intervals are: (1) the reciprocal integration interval which is defined as $r_{1}=1 / r_{2} ;(2)$ the half-power point which surrounds the known maximum response frequency. This option can only be used for very smooth PSD functions; (3) arbitrary integration interval $\left(r_{1}, r_{2}\right)$ which surround the maximum response frequency. This option is suitable for those PSD functions which have respective properties.

Since the natural frequency $\omega_{n}$ is unknown. The maximum response frequency $\omega_{\max }$ is used to evaluate $r_{1}$ and $r_{2}$. Thus the estimates of $r_{1}$ and $r_{2}$ are given by

$$
r_{1}=\frac{\omega_{a}}{\omega_{\max }}=\frac{\omega_{a}}{\omega_{n} \sqrt{\left(1-2 \beta^{2}\right)}}, \quad r_{2}=\frac{\omega_{b}}{\omega_{\max }}=\frac{\omega_{b}}{\omega_{n} \sqrt{ }\left(1-2 \beta^{2}\right)} .
$$

The solution $\beta$ of $F(\beta)$ can be solved by Newton-Raphson method or false position method.

The traditional half-power method for a small damping ratio is given by the following

$$
\omega_{+1 / 2}-\omega_{-1 / 2}=2 \beta \omega_{n}, \quad \omega_{-1 / 2}<\omega_{\max }<\omega_{+1 / 2}
$$

where $\omega_{+1 / 2}, \omega_{-1 / 2}$ are the nearest frequencies to $\omega_{\max }$ and have the property $S\left(\omega_{-1 / 2}\right)=$ $S\left(\omega_{+1 / 2}\right)=\frac{1}{2} S\left(\omega_{\max }\right)$. The estimated $\beta$ can be obtained from the above formula.

\section{SIMULATIONS AND RESULTS}

In this section we will simulate the responses of a sdof and a 2 dof dynamic system. We calculate the spectral shapes of the response by using the three spectral methods, 

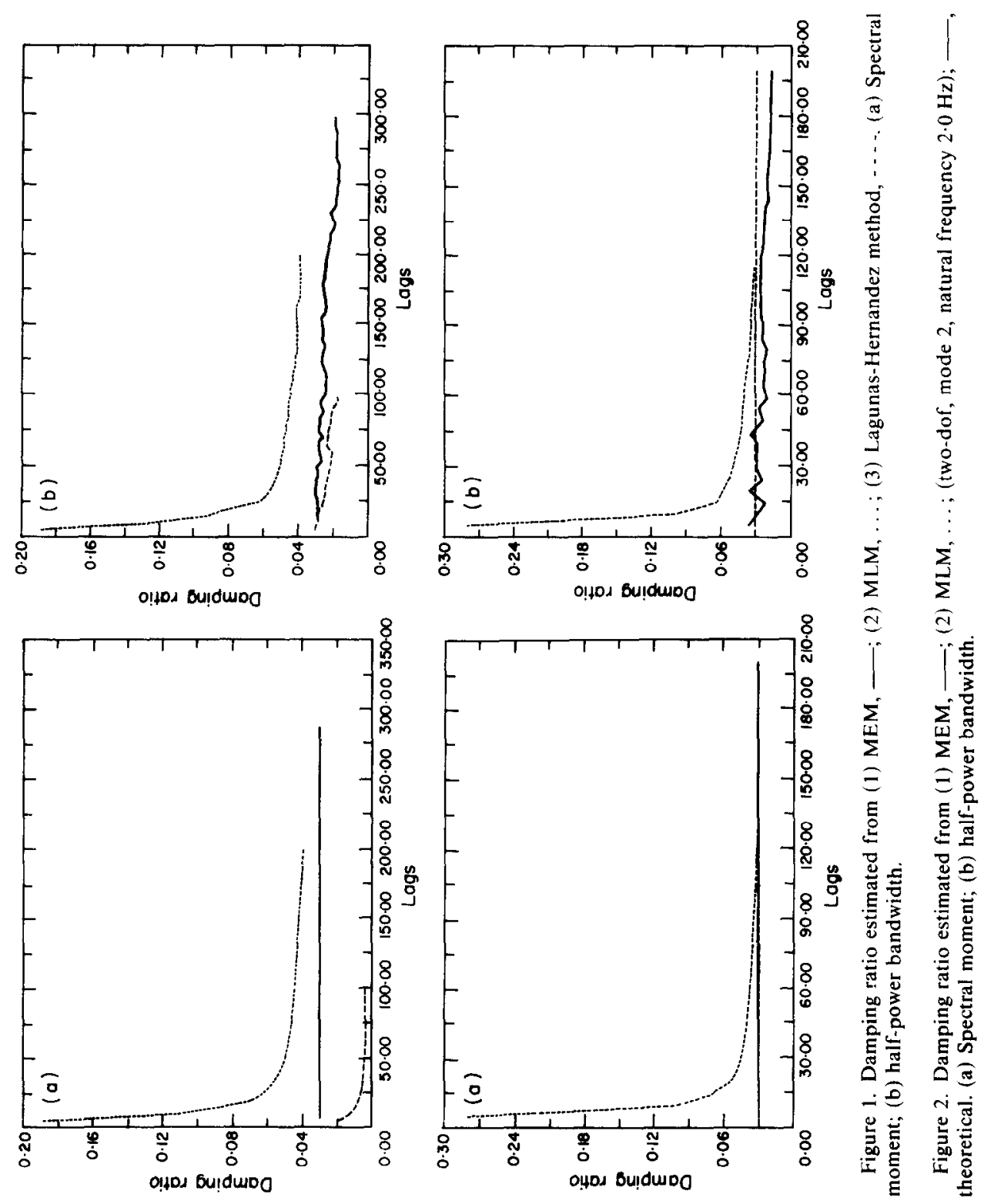
then apply the iterative method and half-power method to estimate the damping ratio and natural frequency. The dynamics of the sdof system in our simulation is

$$
\frac{\mathrm{d}^{2} x(t)}{\mathrm{d} t^{2}}+2 \beta \omega_{n} \frac{\mathrm{d} x(t)}{\mathrm{d} t}+\omega_{n}^{2} x(t)=\omega_{n}^{2} x(t), \quad x(0)=\frac{\mathrm{d} x(0)}{\mathrm{d} t}=0 .
$$

The discrete system which is analogous with the above continuous system is [13]

$$
x(n)=A x(n-1)-B x(n-2)+(1-A+B) u(n)
$$

where

$$
A=2 \mathrm{e}^{-\beta \omega_{n} \Delta} \cos \left\{\omega_{n} \Delta\left(1-\beta^{2}\right)^{1 / 2}\right\}, \quad B=\mathrm{e}^{-2 \beta \omega_{n} \Delta}
$$

$u(n)$ is the Gaussian white noise input at discrete time $n \Delta, \Delta=0.1 \mathrm{sec}$, and $\omega_{n}=$ $2 \pi \cdot(1 \cdot 0 \mathrm{~Hz})$. This formula is obtained by a technique which is called discrete analog system synthesis in [12]. The same technique is also applied to simulating a 2 dof system with two natural frequencies $\left\{\omega_{n}^{1}=2 \pi \cdot(1 \cdot 0 \mathrm{~Hz}), \omega_{n}^{2}=2 \pi \cdot(2 \cdot 0 \mathrm{~Hz})\right\}$ and two modal damping ratios $\left\{\beta^{1}=0.03, \beta^{2}=0.03\right\}$. The transient responses are truncated by inspecting the response graphs. The stationary responses are used for calculating the autocorrelation functions. The total of $12 \times 1024$ sampled data $(20.48 \mathrm{~min})$ are used for calculating the autocorrelation functions $R_{x}(m)=R_{x}(-m)$ [5], where $m=0,1,2, \ldots, M$ is the discrete lag as before.

There is an undetermined parameter, order $M$, in the three spectral methods. In order to signify its importance in the estimation, we include this parameter as another dimension in the estimation.

The results of the sdof system are plotted in Figs 1(a) and (b). The results of the 2 dof system are plotted in Figs $2(\mathrm{a})$ and (b) for the mode at natural frequency $2 \mathrm{~Hz}$. All these figures are plotted with the variable lags $M$. The curves in the figures are truncated at a specific $M$ where the limitations of that spectral method is met by using the calculated $R_{x}(m), m=0,1,2, \ldots, M$, which have error variances. The curves in each figure display the general performances of the specific combination.

\section{SUMMARY AND CONCLUSIONS}

Two methods (the iterative method and the half-power bandwidth method) with three kinds of estimated PSD functions (MLM, MEM, and the Lagunas-Hernandez method) have been presented to estimate the natural frequency and equivalent damping ratio of a dynamic system. The four graphs are representative cases in our experiences, there are several important features on these graphs. In order to examine the effect of finite length data in the estimation, we have been using the theoretical autocorrelation functions given in equation (1) instead of the estimated values of equation (2) to calculate the three spectra and the damping ratio. The results are almost identical to those obtained in equation (2). This is because in equation (2) we use very long data length $(12 \times 1024)$, this means that our procedures are applicable to short data length and noisy records.

The MEM have several other algorithms [4], we have used only equation (3) as a representative of the MEM in our estimation as the results obtained are comparable, see $[7,8]$.

Among all combinations in our test the estimation of the damping ratio using equations (2), (3), and (6) has the best result. This can be seen from the Figs 1(a) and 2(a). The estimated damping ratios by the MEM and spectral moments almost coincide with the true value of 0.03 . 
The Lagunas-Hernandez method is not capable of estimating the damping ratio, this can be seen in Fig. 1(b). The spectrum of Lagunas-Hernandez is very unstable and its spectral envelope cannot be used for damping ratio estimation.

The estimation by the MLM and equation (6) is good, it converges asymptotically to the true value and serves as the upper bound of the estimation, and so, we can use it to confirm the estimated damping ratio.

The estimation by the half power bandwidth (7) is shown to be somewhat unstable when Figs 1(b) and 2(b) are compared with Figs 1(a) and 2(a).

Figures 1 and 2 show only some of our results, many other examples from our simulations and measurements support these conclusions, e.g. analyses of the random vibrations of a single production platform in the Gulf of Mexico [8].

\section{REFERENCES}

1. L. MeIrovitCH 1967 Analytical Methods in Vibrations. New York: Macmillan.

2. D. J. EwINS 1980 Modal Testing: Theory and Practice. Taunton: Research Studies Press.

3. A. D. NASHIF and D. G. Jones (1985) Vibration Damping. New York.

4. S. HAYKIN ed. 1979 Nonlinear Methods of Spectral Analysis, Topics in Applied Physics, Vol. 34. Berlin: Springer-Verlag.

5. A. V. OpPENHEIM and R. W. SChatrer 1975 Digital Signal Processing. Englewood Cliffs, NJ: Prentice-Hall.

6. R. T. LACOSs 1971 Geophysics, 36, 661-675. Data adaptive spectral analysis methods.

7. P. F. Fougere 1987 International Conference on Acoustics, Speech, and Signal Processing, 9.8, pp. 352-355. On the extreme accuracy of maximum entropy spectrum estimation from an error-free autocorrelation function.

8. M. F. COOK 1982 Dissertation, Master of Science, Department of Ocean Engineering, Massachusetts Institute of Technology. Damping estimation response prediction and fatigue calculation of an operational single pile platform.

9. M. A. Lagunas-Hernandez and A. Gasull-Llampallas 1984 IEEE Transactions on Acoustics, Speech and Signal Processing, 32, 170-173. An improved maximum likelihood method for power spectral density estimation.

10. S. H. CRANDAlL and W. D. MARK 1963 Random Vibration in Mechanical Systems. New York: Academic Press.

11. N. C. NIGAM 1983 Introduction to Random Vibration. Cambridge, MA: MIT Press.

12. J. M. SMITH 1977 Mathematical Modelling and Digital Simulation for Engineers and Scientists. New York: Wiley-Interscience.

13. E. H. Vanmarcke 1977 Stochastic Problems in Dynamics, B. L. Clarkson (ed.). Method of spectral moments to estimate structural damping. London: Pitman.

14. S. Shyam Sunder, S. E. Grewatz, and S. K. Ting 1985 Structure Safety, 3, 1-11. Modal identification using spectral moments.

15. S. Shyam Sunder and R. A. SANNi 1984 Journal of Applied Ocean Research. Foundation stiffness identification for offshore platforms. 